\title{
Timing of apoptosis onset depends on cell cycle progression in peripheral blood lymphocytes and lymphocytic leukemia cells
}

\author{
YONGDONG FENG $^{1 *}$, JIANHONG WU $^{1 *}$, XIAOLAN FENG ${ }^{1}$, DEDING TAO $^{1}$, JUNBO HU $^{1}$, JICHAO QIN $^{1}$, \\ XIAOLAN LI ${ }^{1}$, WEI XIAO ${ }^{1}$, KEVIN GARDNER ${ }^{2}$, SUSAN I.V. JUDGE ${ }^{3}$, QINGDI Q. LI $^{2}$ and JIANPING GONG ${ }^{1}$ \\ ${ }^{1}$ Cancer Center, Tongji Hospital, Tongji Medical College, Central China University of Science and Technology, Wuhan 430030, \\ P.R. China; ${ }^{2}$ Laboratory of Receptor Biology and Gene Expression, Center for Cancer Research, National Cancer Institute, \\ National Institutes of Health, Bethesda, MD 20892; ${ }^{3}$ MS Center of Excellence-East, VA Maryland Health Care System, \\ and Department of Neurology, University of Maryland School of Medicine, Baltimore, MD 21201, USA
}

Received February 8, 2007; Accepted March 12, 2007

\begin{abstract}
Apoptosis results in cell death within 10 min after initiation by Bcl-2 family proteins and mitochondria; however, cells enter the apoptotic pathway at different elapsed times after being triggered. Intrinsic factors related to chemical or physical cell damage can initiate apoptosis at a specific cell cycle phase; it is not clear whether cells insulted via an extrinsic pathway also die at a specific cell cycle phase, or how apoptosis is related to cell cycle progression in cells. To illustrate the kinetic changes of apoptosis during cell cycle progression, we examined both intrinsically and extrinsically induced apoptosis in MOLT-4 and Jurkat lymphocytic leukemia cells and in cultured peripheral blood lymphocytes (PBLs) using a recently modified annexin $\mathrm{V}$ and propidium iodide method, which detects cell cycle-specific apoptosis. Apoptosis predominantly occurred at a specific cell cycle phase. Leukemia cells were sensitive to induction by both intrinsic (X-rays, UV light, camptothecin, arsenic trioxide, and the traditional Chinese medicine Jinke, which is an extract of Auricularia auricula) and extrinsic factors (via Fas and TNF receptor pathways). The phase at which leukemia cells entered apoptosis depended on the nature of the insult (X-ray or UV, G1-phase; camptothecin, S-phase; arsenic, G1/S phases; Jinke, G1/S phases; and TNF or Fas
\end{abstract}

Correspondence to: Dr Jianping Gong, Department of Surgery, Tongji Hospital, Tongji Medical College, Wuhan 430030, P.R. China E-mail:.jpgong@tjh.tjmu.edu.cn

Dr Qingdi Quentin Li, Laboratory of Receptor Biology and Gene Expression, National Cancer Institute, National Institutes of Health, Building 41, Room D305, Bethesda, MD 20892, USA

E-mail: liquenti@mail.nih.gov

${ }^{*}$ Contributed equally

Key words: apoptosis, cell cycle phase specificity, apoptotic pattern, lymphocytes, leukemia cells, Chinese medicine, Jinke ligand, G1/S phases), whereas PBLs did not exhibit such insultdependent differences. PHA-stimulated PBLs entered apoptosis, and additional cells were recruited following additional insults. Unstimulated PBLs remained unresponsive to apoptosis, and proliferating cells became insensitive to the insults after the cell cycle checkpoint was abolished by caffeine. Confluent or starving PBLs were also unresponsive to apoptotic triggers. Thus, apoptotic cell death is a cell cycle event with most, if not all, apoptosis being initiated during a particular cell cycle phase, and changes in the cell cycle result in changes in the apoptotic pattern and schedule. The coordination of apoptosis and proliferation in cells offers a mechanism for the integration of both cell cycle and apoptotic signals.

\section{Introduction}

Apoptosis is a structurally distinct mode of cell death that is morphologically characterized by cell shrinkage accompanied by transient but violent surface bubbling and blebbing, ultimately resulting in the separation of the cell into a cluster of membrane-bound bodies. Molecular studies on apoptosis have led to the discovery of a set of proteins that regulate the cellular commitment to death; among these proteins are the Bcl-2 family modulators and caspases. Two main signal transduction pathways are involved in apoptosis, the mitochondrial and death receptor pathways. Recent studies have shown that key cell cycle regulators participate in the apoptotic process, suggesting that apoptosis is a cell cycle event and might not occur in the absence of cell cycle progression. Both the apoptotic and cell cycle processes are of widespread biological importance owing to their influence on development, differentiation, homeostasis, and the regulation and function of the immune system $(1,2)$. The two processes are necessarily coupled in determining the life and death of cells (3).

As an integral part of life, the apoptotic process, from the initial trigger to the destruction of the cell, can take hours or even days. However, only approximately $10 \mathrm{~min}$ are required for the execution stage of apoptosis, which begins with the permeabilization of the mitochondrial outer membrane by proapoptotic members of the Bcl-2 family and the release of inner membrane proteins into the cytosol to activate caspases 
(4). Little is known about how and when living cells are transformed to begin this crucial 10 min, or why Bcl-2 family proteins are activated at a particular time, which may be hours or days after a cell has been triggered to die. Many cancerspecific treatments intended to induce tumor cell apoptosis by radiotherapeutic or chemotherapeutic methods are based on the assumption that malignant cells have a greater tendency to die as a result of their higher proliferation index. Nevertheless, it is difficult to predict treatment outcomes because little is known about the time lag between treatment and the initiation of apoptosis in a living cell. The diversity of the apoptotic process is attributable to many different variables, including the genetic background of a cell and the cellular context.

Although chemical or physical damage to a cell can occur at a specific cell cycle phase, it is not clear why faster-growing tumor cells are more sensitive to chemotherapy or physiotherapy, or whether damaged cells always die at a specific cell cycle phase. Additional unresolved issues include the mechanism determining the cell cycle-specific pattern of apoptosis and the mechanism by which apoptosis becomes programmed during cell cycle progression. However, it is clear that the two opposing aspects of a cell's fate, the cell cycle and apoptosis, must be closely intertwined. Previous genetic, molecular and morphological studies have separately measured apoptosis and cell cycle progression and thus have limitations with respect to analyzing the dynamic relationship between the two processes.

The development of techniques taking advantage of terminal deoxynucleotidyl transferase (TdT) or annexin V and propidium iodide staining has overcome the inherent limitations of genetic, molecular and morphological techniques by enabling real-time cytometric analysis in order to discriminate viable, apoptotic and necrotic cells during apoptosis and cell cycle progression (5), thus revealing the cell cycle-specific nature of apoptotic processes. These techniques can be used to further elucidate the pattern and program of cell cycle phase-specific apoptosis during cell cycle progression by addressing questions regarding the relationship between the mitochondrial and death receptor pathways, the relative sensitivities of cells at different cell cycle phases, and the variation of apoptosis patterns in cells of different proliferation status.

Cell cycle checkpoints regulate the progression of the cell cycle, and as such they are potential sites of intersection between the cell cycle and cell cycle-specific apoptosis. Treating cells with caffeine, the first drug shown to override cell cycle checkpoints (6-8), could be useful for analyzing the relationship among cell cycle checkpoints, cell cycle progression and cell death.

In this study, to investigate the relationship between apoptosis and cell cycle progression, we examined both intrinsically and extrinsically induced apoptosis in the MOLT-4 and Jurkat leukemia cell lines and in cultured peripheral blood lymphocytes (PBLs) using a recently described annexin V and propidium iodide (API) method, which detects cell cycle phase-specific apoptosis (5). PBLs and leukemia cell lines offer good models for the study of apoptosis during cell cycle progression. PBLs can be kept in quiescence $(\mathrm{G} 0)$ unless stimulated by a mitogen to enter the cell cycle, and unstimulated and stimulated PBLs show different sensitivities to apoptotic insults. In contrast, MOLT-4 and Jurkat cells are always progressing through the cell cycle and can be induced to enter apoptosis, showing good sensitivity to different apoptotic insults.

Here, the cells were cultured in the presence or absence of caffeine and were insulted by treatment with an extrinsic factor, which included Fas ligand (FasL) and tumor necrosis factor (TNF)- $\alpha$ to activate the two respective pathways, or an intrinsic factor, which included the topoisomerase I inhibitor camptothecin (CPT), different X-ray dosages, and the traditional Chinese medicine Huaier, which is prepared as an extract of the plant Auricularia auricula and is commercially available in China under the trade name Jinke. We found that the onset of apoptosis varied depending on cell cycle progression in cultured PBLs and in the two leukemia cell lines and that cultured cells irradiated in the presence of caffeine showed resistance to apoptosis and continued to progress through the cell cycle. These results demonstrate that apoptotic cell death is a cell cycle event and that most, if not all, apoptosis is initiated during specific cell cycle phases, necessitating the coordinated integration of both cell cycle and apoptotic signals in the cell.

\section{Materials and methods}

Cells and cell culture. MOLT-4 and Jurkat cells (American Type Culture Collection, Manassas, VA, USA) were maintained in RPMI-1640 medium (Gibco, Grand Island, NY, USA) supplemented with $10 \%$ fetal calf serum (Gibco) and 2 mM L-glutamine (Sigma Chemical Co., St. Louis, MO, USA). The experiments were performed on cells during the exponential growth phase, as described elsewhere (5).

Blood was obtained by venipuncture from healthy volunteers or patients, and human peripheral blood lymphocytes (PBLs) were isolated by density gradient centrifugation. The cells were washed twice with phosphate-buffered saline (PBS), resuspended at a density of $10^{6}$ cells/ml in RPMI-1640 medium supplemented with $10 \%$ fetal calf serum and antibiotics, and cultured in the presence or absence of phytohemagglutinin (PHA) (Sigma), unless otherwise indicated.

Drugs and cell treatments. Exponentially growing MOLT-4 cells were exposed to $2 \mathrm{~Gy}$ X-ray irradiation for $9 \mathrm{~h}$, or exposed to $10 \mathrm{~Gy} \mathrm{X}$-ray irradiation for $3 \mathrm{~h}$. The cells were harvested and subjected to an API assay. For treatment with ultraviolet (UV) irradiation, flasks containing MOLT-4 cells were placed directly on the glass surface of a mini-transilluminator $\left(0.375 \mathrm{w} / \mathrm{cm}^{2}\right.$; Bio-Rad, Hercules, CA, USA $)$, illuminated with UV light for $40 \mathrm{sec}\left(150 \mathrm{kj} / \mathrm{m}^{2}\right)$, and subsequently incubated at $37^{\circ} \mathrm{C}$ for $4 \mathrm{~h}$ before an API assay was performed.

A stock solution of camptothecin (CPT; Sigma) was prepared at a concentration of $200 \mu \mathrm{g} / \mathrm{ml}$ in dimethyl sulfoxide (DMSO). Each agent was diluted separately with RPMI-1640 medium to the following final respective concentrations: $0.15 \mu \mathrm{M} \mathrm{CPT}, 1 \mu \mathrm{M}$ arsenic trioxide (Sigma), $3.0 \mathrm{~g} / \mathrm{l}$ of Jinke, $1 \mathrm{mg} / \mathrm{l}$ of mouse anti-human Fas antibody (Pharmingen), and $40 \mu \mathrm{g} / \mathrm{l}$ of tumor necrosis factor (TNF)- $\alpha$ (Pharmingen).

MOLT-4 cells were incubated in the presence of CPT for $4 \mathrm{~h}$, in the presence of arsenate for $36 \mathrm{~h}$, or in the presence of 
the Chinese medicine Jinke for $16 \mathrm{~h}$ and then harvested for API assays. Exponentially growing Jurkat cells were incubated in the presence of Fas antibody for $36 \mathrm{~h}$, in the presence of TNF- $\alpha$ for $30 \mathrm{~h}$, or in the presence of Jinke for $16 \mathrm{~h}$ and then harvested for API assays.

The PBLs were incubated with phytohemagglutinin (PHA; Sigma) at a final concentration of $10 \mu \mathrm{g} / \mathrm{ml}$ for $24 \mathrm{~h}$ and then further cultured for $4 \mathrm{~h}$ in the presence of CPT or after exposure to 10 Gy X-ray irradiation. Caffeine at a dose of $50 \mathrm{mM}$ was added to the PBLs during PHA stimulation or CPT treatment, respectively, and the cells were irradiated. The cells were subjected to analysis at the indicated culture times.

Apoptosis detected by the annexin $V$ and propidium iodide (API) method. Fluorescein isothiocyanate (FITC)-conjugated annexin $\mathrm{V}$ and propidium iodide (PI) cell staining coupled with DNA content analysis was used to measure the cell cycle phase specificity of apoptosis (5). First, aliquots of freshly collected cells were suspended in phosphate-buffered saline (PBS), collected by centrifugation at $200 \mathrm{x}$ g for $5 \mathrm{~min}$, and resuspended at a density of approximately $10^{6} \mathrm{cell} / \mathrm{s} / \mathrm{ml}$ in binding buffer, pH 7.4, containing $10 \mathrm{mM}$ Hepes (Sigma), $140 \mathrm{mM}$ $\mathrm{NaCl}$, and $2.5 \mathrm{mM} \mathrm{CaCl}_{2}$. For the detection of total apoptosis, $5 \mu \mathrm{l}$ of FITC-annexin V (Pharmingen, San Diego, CA) and $10 \mu 1$ of PI (Sigma) at a concentration of $50 \mu \mathrm{g} / \mathrm{ml}$ in PBS were added to the cells, and the cells were incubated for $20 \mathrm{~min}$ at room temperature in the dark. For the API method, $5 \mu 1$ of FITC-annexin V was added to $100 \mu 1$ of freshly collected cells in binding buffer, and the cells were incubated in the dark at room temperature for $20 \mathrm{~min}$. The cells were then rinsed in binding buffer, resuspended in $1 \mathrm{ml}$ of $1 \%$ methanol-free formaldehyde in binding buffer, and set on ice for $30 \mathrm{~min}$. The cells were then rinsed twice, resuspended in $0.5 \mathrm{ml}$ of a solution containing $50 \mu \mathrm{g} / \mathrm{ml}$ of PI, $0.1 \%$ RNase A (Sigma), $500 \mu \mathrm{g} / \mathrm{ml}$ of digitonin (Sigma), $10 \mathrm{mM}$ PIPES (Sigma), $2 \mathrm{mM}$ $\mathrm{CaCl}_{2}$, and $0.1 \mathrm{M} \mathrm{NaCl}$, and placed at room temperature for $1 \mathrm{~h}$ in the dark. The cell cycle phase specificity of apoptotic cells was analyzed by flow cytometry. Untreated cells served as the negative controls. Cell fluorescence was measured using a FACSort flow cytometer (Becton-Dickinson, San Jose, CA, USA). CellQuest software was used for the acquisition and analysis of data.

Statistical analysis of data. The quantitative experiments were analyzed by Student's t-test. Values of $\mathrm{P}<0.05$ with a two-sided test were considered significant.

\section{Results}

Cell cycle phase specificity of apoptosis induced by intrinsic and extrinsic factors. A number of different factors can induce apoptosis; these include intrinsic factors such as chemical agents (e.g., CPT and arsenic trioxide), some Chinese medicines (e.g., Jinke), and treatments that cause physical damage (e.g., X-rays and UV irradiation), as well as extrinsic factors that activate certain receptor pathways (e.g., TNF and Fas ligand). Given that apoptosis was shown to be specified at a particular phase of the cell cycle in some cases (5), we investigated whether apoptosis induced by these factors
A

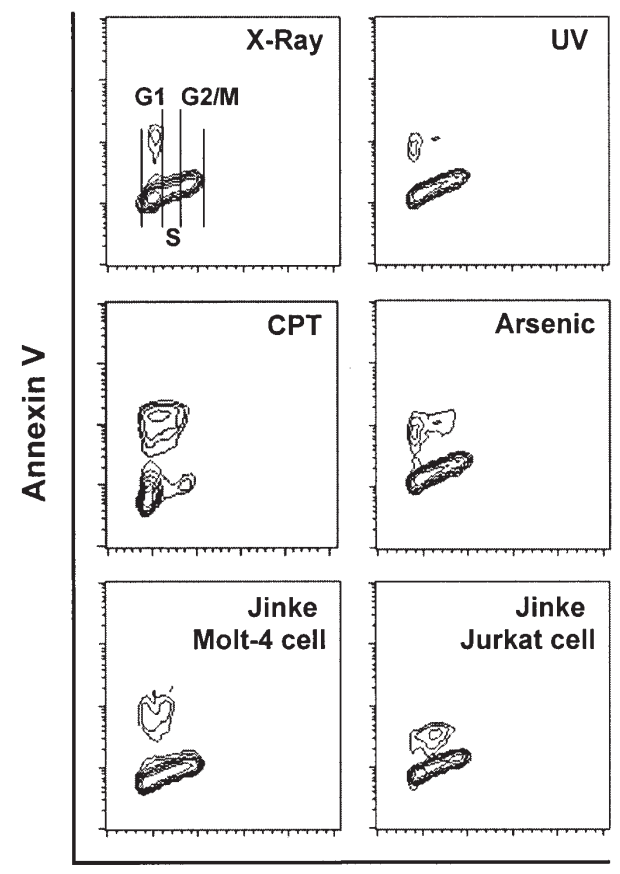

DNA Content

B

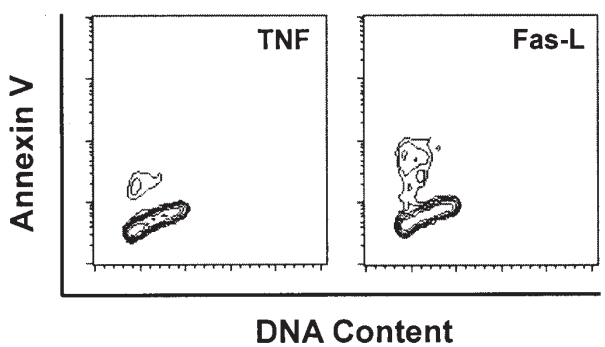

Figure 1. Differences in cell cycle phase specificity of apoptosis in response to various inducers. (A) Apoptosis was induced by intrinsic factors and was measured by the annexin V and propidium iodide method. MOLT-4 and Jurkat cells show similar patterns of apoptosis, except when induced by Jinke. DNA damage produced by irradiation with 10 Gy X-rays or UV light results in G1-phase apoptosis. Camptothecin (CPT) induces S-phase apoptosis, and arsenic trioxide causes cells in G1/S phases to enter apoptosis. The Chinese medicine Jinke induces apoptosis at $\mathrm{S}$ phase in MOLT-4 cells but at G1/S phases in Jurkat cells. (B) Apoptosis induced via the Fas or tumor necrosis factor (TNF) receptor pathway shows G1/S phase specificity in MOLT-4 and Jurkat lymphocytic leukemia cells.

was initiated at a specific phase of the cell cycle, using a newly established API method to simultaneously measure the apoptosis rate and DNA ploidy (5). As shown in Fig. 1A, the DNA damage produced by irradiation with UV light or $10 \mathrm{~Gy}$ $\mathrm{X}$-rays induced apoptosis exclusively at G1 phase in MOLT-4 and Jurkat cells. CPT was highly selective in inducing S phasespecific apoptosis, whereas toxic arsenic trioxide induced apoptosis in a G1/S pattern in these two cell lines. The Chinese medicine Jinke induced two different patterns of cell cyclespecific apoptosis, $\mathrm{S}$ phase-specific in MOLT-4 cells and a G1/S pattern in Jurkat cells. The activation of the cell surface TNF receptor or Fas pathway caused most cells to die according 
A

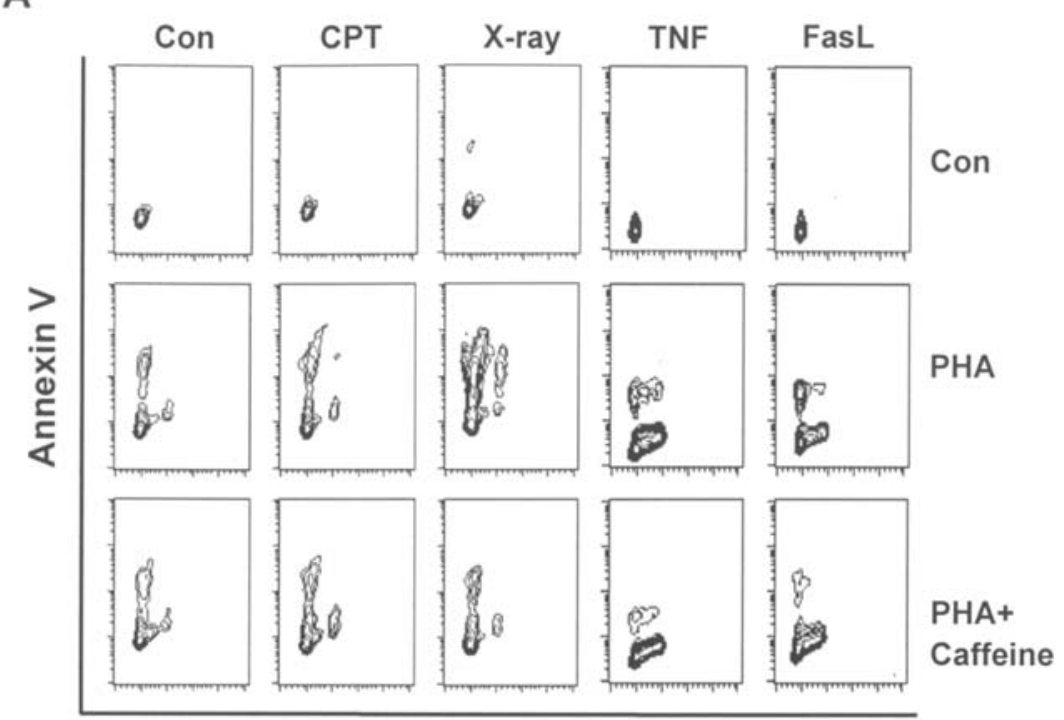

DNA Content

B

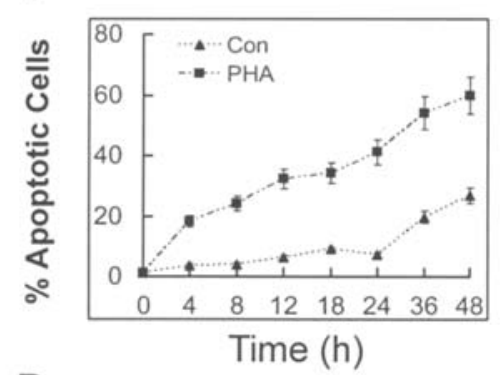

$\mathrm{D}$

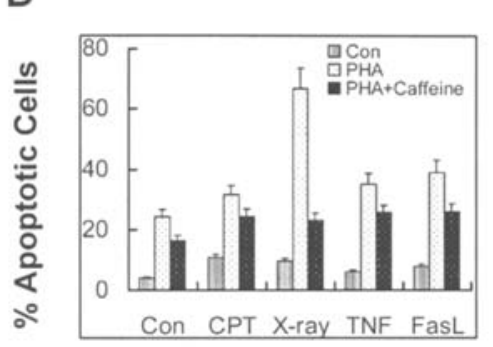

C

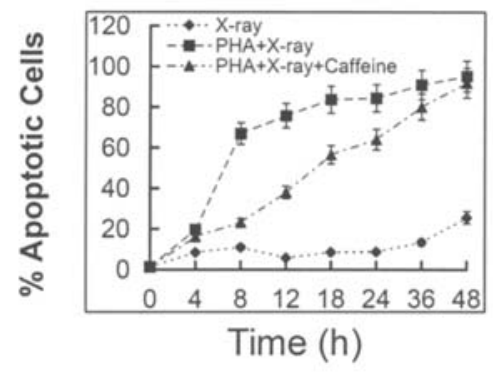

Figure 2. Apoptosis and cell cycle progression in peripheral blood lymphocytes (PBLs). (A) The apoptosis pattern was measured by the annexin V and PI method. Unstimulated PBLs are unresponsive to apoptosis in the presence or absence of intrinsic (CPT and X-rays) or extrinsic (TNF and FasL) apoptosis factors (upper panel). PBLs become apoptotic following phytohemagglutinin (PHA) stimulation for $8 \mathrm{~h}$, with more cells entering apoptosis upon induction by intrinsic or extrinsic factors (middle panel). Caffeine suppresses cell apoptosis (lower panel). (B and C) PHA-stimulated PBLs become apoptotic upon the application of intrinsic and extrinsic factors, as a function of time, while the application of caffeine reduces apoptotic rates. (D) The apoptotic rate at $8 \mathrm{~h}$ following treatment with PHA and caffeine is the same in response to all of the apoptosis factors examined in PBLs. Con, control.

to a G1/S pattern (Fig. 1B). Thus most, if not all, apoptosis exhibited cell cycle phase specificity, and cell cycle phasespecific apoptosis differed with cell type and stimulant. Regardless of the apoptosis pattern exhibited, our results demonstrate that apoptosis is a cell cycle phase-selective or cell cycleregulated event.

G0 cells are insensitive but proliferating cells are sensitive to apoptotic triggers. In the leukemia cell lines, apoptosis was intertwined with the progression of the cell cycle; we also examined this relationship in normal PBLs. As shown in Fig. 2A, intact cultured human PBLs, which were in G0, were primarily unresponsive to apoptosis induced by either intrinsic or extrinsic factors, although a few cells did exhibit apoptosis (Fig. 2A, upper panel). Thus, quiescent G0 cells appear to be resistant to apoptosis or to have a blunted response.

PHA-stimulated PBLs, which entered the cell cycle, exhibited increased apoptosis during G1 phase [Fig. 2A (middle panel) and B]. In addition, proliferating PBLs were more sensitive to intrinsic and extrinsic apoptotic insults, in comparison with unstimulated PBLs [Fig. 2A (middle panel) and D]. The apoptosis rate increased to $31.56 \%$ during the G1/S phases when stimulated by CPT and to $66.89 \%$ during the G1-G2/M phases following X-ray irradiation. 
A

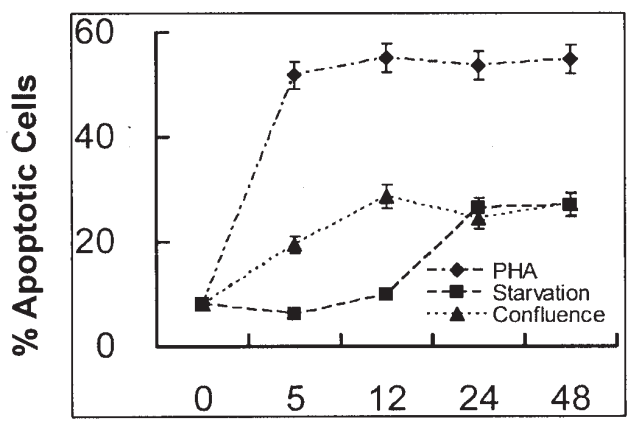

Time (h)

B

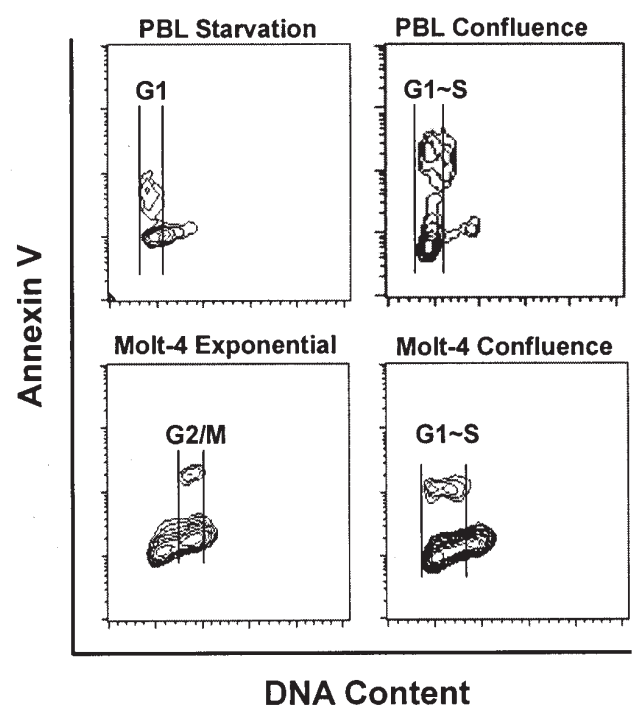

Figure 3. Patterns of apoptosis under different growth states. (A) The apoptotic rate was higher in exponentially growing (PHA) PBLs than in confluent (Confluence) or starved (Starvation) PBLs in the presence of PHA. (B) The cell cycle phase specificity of apoptosis also varies among the different growth states in PBLs and MOLT-4 lymphocytic leukemia cells

After the checkpoint was abolished, induced cells avoided apoptosis. The addition of caffeine has been shown to abolish cell cycle checkpoints, allowing cells to progress through the cell cycle. As seen in Fig. 2A (lower panel), C and D, the presence of caffeine caused a reduction in the apoptosis rate after PHA stimulation in the presence and absence of additional apoptotic factors. Thus, a cell cycle checkpoint might provide a point of coupling between apoptosis and the cell cycle. However, the effects of caffeine were ambiguous, reflecting the complexity of the checking mechanism in cell cycle progression.

Cells at different growth states exhibit different patterns of apoptosis. Cells growing under different conditions, e.g., starvation or confluency, exhibit different cell cycle progression rates $(9,10)$. PHA-stimulated PBLs, either confluent or starved, exhibited lower apoptosis rates in comparison with the rates of exponentially growing cells (Fig. 3A). In starved cells, apoptosis occurred exclusively in G1 phase, whereas apoptosis occurred during G1/S in confluent cells (Fig. 3B, upper panel).
A

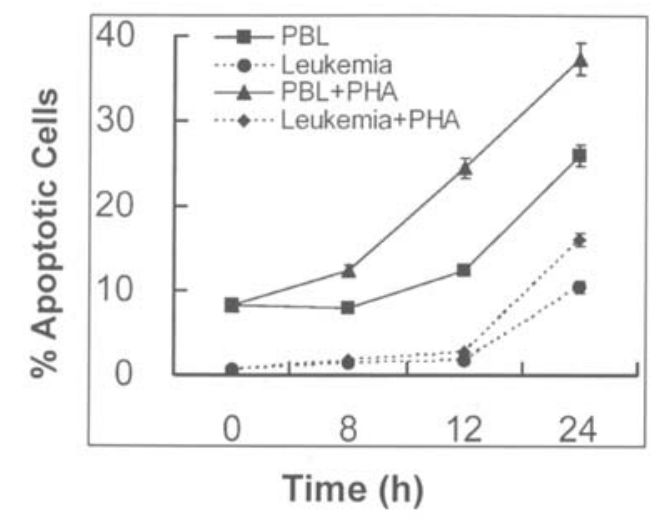

B

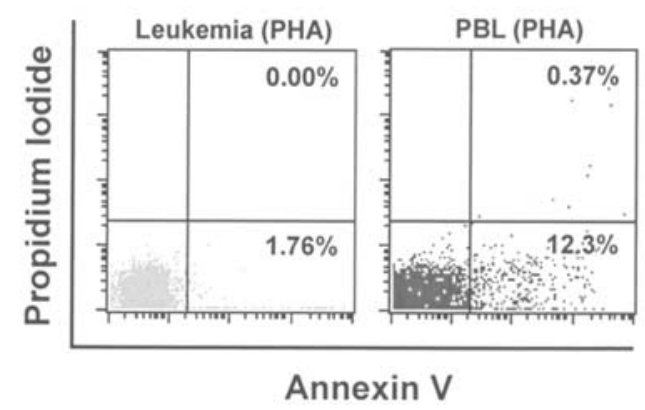

Figure 4. In comparison with normal PBLs, human leukemia cells were unresponsive to the mitogen PHA induction of apoptosis. Apoptotic cells were assessed by the annexin V and PI method. (A) The apoptotic rate is lower in PHA-stimulated human leukemia cells than in PHA-stimulated PBLs from healthy volunteers. (B) A dot plot shows the detection of apoptotic cells after stimulation of normal PBLs and leukemia cells with PHA for $8 \mathrm{~h}$.

MOLT-4 cells also showed growth state-dependent apoptosis. With induction by 2 Gy X-ray irradiation, exponentially growing MOLT-4 cells entered apoptosis at G2/M phase, whereas confluent MOLT-4 cells $\left(1 \times 10^{7}\right.$ cells $\left./ \mathrm{ml}\right)$ showed a G1/S pattern of apoptosis (Fig. 3B, lower panel).

Difference in the response to PHA stimulation between PBLs and leukemia cells from patients. The apoptotic rate in PHA-stimulated normal PBLs was greater than that in PHAstimulated leukemia cells from patients (Fig. 4), revealing the uncoupling of apoptosis from the cell cycle progression in leukemia cells.

\section{Discussion}

There has been great progress in understanding cell proliferation and cell death during the last century, and the cell cycle and apoptosis have become extremely popular topics in the life sciences. Many studies have focused on the relationship between the cell cycle and apoptosis because both processes interact to maintain homeostasis in cells and organisms (11-14). Although the coordination of apoptosis and proliferation (CAP) has attracted much attention, the exact definition and major principles of CAP are not certain.

The basic pathway of apoptosis in vertebrates begins with the permeabilization of the mitochondrial outer membrane by 
proapoptotic members of the $\mathrm{Bcl}-2$ family, resulting in a release of proteins from the inner membrane space into the cytosol. The inevitable process, beginning with the first mitochondrial changes and culminating in the activation of caspases, usually takes approximately $10 \mathrm{~min}$; however, the entire course, from the initial trigger to the destruction of the cell, can take hours or even days. It remains unclear whether or when a cell will begin this crucial 10-min process and why mitochondrial changes and the subsequent consequences occur at a particular time, hours or days after a cell has been triggered to die. Nevertheless, the specific cell cycle phase at which apoptosis is initialized can be determined (15-16). Analyzing cell cycle phase-specific apoptosis provides an approach to addressing the remaining questions regarding CAP.

Cell cycle phase specificity of apoptosis. To induce apoptosis, we used physical damage, chemicals, toxic agents, and a Chinese medicine as intrinsic factors, and Fas and TNF receptor pathways as extrinsic factors. We showed that apoptosis from both intrinsic and extrinsic pathways exhibited cell cycle phase specificity and that the pattern of apoptosis varied with cell type and the induction agent. Given that our experiments probably included all routes for apoptosis, we concluded that most, if not all, apoptosis is cell cycle phase specific and that apoptosis is a cell cycle event. Thus, the initial time of apoptosis depended on cell cycle progression. Treated cells died at a particular phase of the cell cycle, or cells that were treated at a particular cell cycle phase died. Thus, the cell cycle engine must play a major role in determining the execution stage of apoptosis. The cell cycle phase specificity represents the exact time of the initiation of apoptosis during cell cycle progression. The phase specificity of apoptosis might represent the point at which the accumulated cell damage overwhelms the driving force for proliferation in a cell, and the cell consequently enters apoptosis. This implies that, at this point, the cell cycle machinery and the apoptotic process are acting at cross purposes (17).

Cell cycle progression is necessary for entering apoptosis: the 'no cycle, no apoptosis' and 'first growth, then death' properties. In the human body, the PBL pool is maintained at a steady level unless the body is challenged by environmental stress, in which case the PBL pool is augmented to counteract inflammation or other stress and is then returned to a normal level by widespread apoptosis. Thus, the coordination of apoptosis and proliferation plays an important role in maintaining the PBL pool. The resistance to apoptosis observed in quiescent PBLs in the present study implies that cells not entering the cell cycle do not initiate apoptosis, a property referred to here as 'no cycle, no apoptosis'. Although this concept might oversimplify the actual situation, it has nevertheless been exploited for the treatment of tumors because it causes proliferating tumor cells to be more sensitive to toxic chemicals than normal somatic cells. This property also suggests that pushing quiescent cancer cells into the cell cycle may be a new strategy for treating tumors.

In support of the 'no cycle, no apoptosis' property, PHAstimulated PBLs, which entered the cell cycle, exhibited an increased apoptosis rate in G1 phase and were more sensitive to apoptotic insults than unstimulated cultured PBLs, indicating a coupling of proliferation and apoptosis. In addition, cultured PBLs in a confluent or starving state showed a lower apoptosis rate after stimulation with PHA than PBLs growing under less dense conditions. Given that confluent or starving cells always slow down or stop proliferating (18-21), the reduced rate of apoptosis in these PBLs further supports the proposition that cells must first begin proliferating before they can undergo apoptosis, which we refer to as 'first growth, then death'.

This relationship between cell growth and cell death has been thought of as a built-in failsafe mechanism to curtail inappropriate proliferation of somatic cells $(3,22)$. The coupling of apoptosis and proliferation during the cell cycle could serve to maintain cellular homeostasis in the body, not only with respect to hyperplasia but also in terms of tumors, in which apoptosis has become uncoupled from proliferation. Thus, the balance between cellular life and death would be determined by the complicated context of survival and death signals both inside and outside the cell.

Cell cycle checkpoints are necessary for entering apoptosis: the 'no stop, no death' and 'first stop, then death" properties. Cell cycle checkpoints serve as negative regulators to enforce the order of cell cycle events and to provide optimal timing of phase transitions $(6,23-25)$. The addition of caffeine abolishes the checkpoints and allows cells to advance through the cell cycle. In the present study, abolishing the checkpoints by adding caffeine resulted in reduced rates of apoptosis, indicating that cells continuing through the cell cycle without stopping at checkpoints did not enter apoptosis. This is referred to here as the 'no stop, no death' property. Cell cycle checkpoints may represent the point at which proliferation and apoptosis intersect, providing an opportunity for all positive and negative signals to be integrated in determining whether the cell will live or die. Thus, the time interval between the apoptotic trigger and the initialization of apoptosis depends not only on cell cycle progression but also on the cell cycle pauses at checkpoints. The role of cell cycle checkpoints in this regard warrants further study.

Apoptosis pattern varies with changes in the cell cycle. After establishing that the initiation of apoptosis depended upon cell cycle progression and checkpoints, we tested whether different growth rates could affect the pattern of apoptosis. In both PBLs and the leukemia cell lines, cells at different growth states exhibited different cell cycle phase specificities and rates of apoptosis. The different growth states mimicked the different proliferation potentials of cells in the body. In the body, proliferating tissues, both cancerous and non-cancerous, are easily damaged by treatments such as tumor chemotherapy. Identifying the cell cycle phase specificity of apoptosis in tumor cells may enable the development of a pharmacological agent that is more specific to cancer cells, thus sparing normal tissues. In addition, as some tumors become chemoresistant, changing the cell cycle progression pattern in the tumor cells may re-establish sensitivity to chemotherapy and reverse the drug resistance.

The molecular mechanisms responsible for the cell cycle phase specificity of apoptosis must involve gene networks or protein interactions. The activation of oncogenes such as $c$-myc, ras or $c$-jun has been shown to trigger, or sensitize a 
cell to, apoptosis $(26,27)$. Wu et al and Harvey et al recently described the gene network of hippo/salvador/warts; these genes are tightly coordinated to regulate either proliferation or apoptosis in the perfection of the fly eye (28). The p53 protein, which acts as a guardian of the genome, is the core of a large protein network that functions at the $\mathrm{G} 1$ or $\mathrm{G} 2 / \mathrm{M}$ checkpoint $(29,30)$. A number of studies suggest that the coordination of apoptosis and proliferation (CAP) has been conserved during eukaryotic evolution as a mechanism for determining the initiation of apoptosis. The progression of the cell cycle and the execution of apoptosis have been demonstrated to be opposing processes (31-33). We found that the unscheduled activation of cyclin-dependent protein kinase 1 (CDK1/CDC2) was involved in the execution stage of apoptosis in MOLT-4 cells induced by X-ray irradiation (17). Others have shown that sublethal damage leading to a small quantity of caspase activation can protect neurons from more severe damage (34). However, much more work is needed to explain the initiation mechanisms of apoptosis with different cell cycle phase specificities.

Deregulated initiation of apoptosis may lead to cancer. The uncoupling of apoptosis and proliferation, causing the deregulated initiation of apoptosis, may lead to the dominance of either proliferation or apoptosis, resulting in tumorigenesis or organ degeneration, respectively. In comparison with normal PBLs, leukemia cells from a patient showed reduced apoptosis when cultured in the presence of PHA, indicating that proliferation dominated over apoptosis in these dysregulated cancerous cells. Given that the cell cycle time is usually normal or even longer than normal in cancer cells (35-37), the recovery of the program of apoptosis would be more crucial than the suppression of proliferation in treating carcinoma cells, which exhibit deregulated coordination of proliferation and apoptosis.

In summary, we demonstrated here that the initial time of apoptosis varied depending on cell cycle progression in cultured PBLs and lymphocytic leukemia cell lines. These results indicate that apoptotic cell death is a cell cycle event and that most, if not all, apoptosis is initiated in a specific cell cycle phase. In addition, our data showed that quiescent G0 cells and cells progressing through the cell cycle without stopping at checkpoints could avoid apoptosis. The apoptotic pattern and schedule varied with changes in the cell cycle, and the uncoupling of apoptosis from cell cycle progression may lead to uncontrolled proliferation. Further studies are needed to investigate the mechanism and timing of the coupling between the cell cycle machinery and the Bcl-2 family proteins to initiate the crucial $10 \mathrm{~min}$ of apoptosis in different normal and tumor cells.

\section{Acknowledgements}

This study was supported by grants from the National Science Foundation of China (nos. 39730270, 30440024 and 30570908), the China State Key Basic Research Program (nos. G1998051212 and 2004CB518705), the Science Foundation of Ministry of Public Health (no. 202-01-06), and the Research Start-up Funds of Ministry of Education for Chinese Overseas Returned Scholars (no. 2003-14), P.R. China.

\section{References}

1. Norbury C and Nurse P: Animal cell cycles and their control. Annu Rev Biochem 61: 441-470, 1992.

2. Chinnaiyan AM and Dixit VM: The cell-death machine. Curr Biol 6: 555-562, 1996.

3. Evan G and Littlewood T: A matter of life and cell death. Science 281: 1317-1322, 1998.

4. Green DR: Apoptotic pathways: ten minutes to dead. Cell 121: 671-674, 2005.

5. Tao D, Wu J, Feng Y and Gong J: New method for the analysis of cell cycle-specific apoptosis. Cytometry 57A: 70-74, 2004.

6. Nurse P: Checkpoint pathways come of age. Cell 91: 865-867, 1997.

7. Moser BA, Brondello JM, Baber-Furnari B and Russell P: Mechanism of caffeine-induced checkpoint override in fission yeast. Mol Cell Biol 20: 4288-4294, 2000.

8. Cortez D: Caffeine inhibits checkpoint responses without inhibiting the ataxia-telangiectasia-mutated (ATM) and ATMand Rad3-related (ATR) protein kinases. J Biol Chem 278: 37139-37145, 2003.

9. Bauer M, Hamm AC and Katzenberger JD: Starvation response in mouse liver shows strong correlation with life-span-prolonging processes. Physiol Genomics 17: 230-244, 2004.

10. Cho SR, Ock SA and Rho GJ: Effects of confluent, roscovitine treatment and serum starvation on the cell-cycle synchronization of bovine fetal fibroblasts. Reprod Domest Anim 40: 171-176, 2005.

11. Hardy K and Stark J: Mathematical models of the balance between apoptosis and proliferation. Apoptosis 7: 373-381, 2002.

12. Fotedar R, Diederich L and Fotedar A: Apoptosis and the cell cycle. Prog Cell Cycle Res 2: 147-163, 1996.

13. Guo M and Hay BA: Cell proliferation and apoptosis. Curr Opin Cell Biol 11: 745-752, 1999

14. King KL and Cidlowski JA: Cell cycle regulation and apoptosis. Annu Rev Physiol 60: 601-617, 1998.

15. Gong J, Li X and Darzynkiewicz Z: Different patterns of apoptosis of HL-60 cells induced by cycloheximide and camptothecin. J Cell Physiol 157: 263-270, 1993.

16. Gorczyca W, Gong J and Darzynkiewicz Z: Detection of DNA strand breaks in individual apoptotic cells by the in situ terminal deoxynucleotidyl transferase and nick translation assays. Cancer Res 53: 1945-1951, 1993.

17. Wu J, Feng Y, Xie D, Tao D, Hu J, Gardner K, Judge S, Li QQ and Gong J: Unscheduled CDK1 activity in G1 phase of the cell cycle triggers apoptosis in X-irradiated lymphocytic leukemia cells. Cell Mol Life Sci 63: 2538-2545, 2006.

18. Martinez-Botas J, Suarez Y, Ferruelo AJ, Gomez-Coronado D and Lasuncion MA: Cholesterol starvation decreases p34(cdc2) kinase activity and arrests the cell cycle at G2. FASEB J 13: 1359-1370, 1999

19. Su SS, Tanaka Y, Samejima I, Tanaka K and Yanagida M: A nitrogen starvation-induced dormant G0 state in fission yeast: the establishment from uncommitted G1 state and its delay for return to proliferation. J Cell Sci 109: 1347-1357, 1996.

20. Wu Y, Pan S, Che S, He G, Nelman-Gonzalez M, Weil MM and Kuang J: Overexpression of Hp95 induces G1 phase arrest in confluent HeLa cells. Differentiation 67: 139-153, 2001.

21. Miloszewska J, Trembacz H and Janik P: Signal transduction in confluent $\mathrm{C} 3 \mathrm{H}$ 10T1/2 cells. The role of focal adhesion kinase. Acta Biochim Pol 48: 175-181, 2001.

22. Harrington EA, Fanidi A and Evan GI: Oncogenes and cell death. Curr Opin Genet Dev 4: 120-129, 1994.

23. Hartwell LH and Weinert TA: Checkpoints: controls that ensure the order of cell cycle events. Science 246: 629-634, 1989.

24. Peeper DS, van der Eb AJ and Zantema A: The G1/S cell-cycle checkpoint in eukaryotic cells. Biochim Biophys Acta 1198: 215-230, 1994.

25. Walworth NC: Cell-cycle checkpoint kinases: checking in on the cell cycle. Curr Opin Cell Biol 12: 697-704, 2000.

26. White DW and Gilmore TD: Transcription factors, oncogenes, and apoptosis. Science 276: 185-186, 1997.

27. Green DR, McGahon A and Martin SJ: Regulation of apoptosis by oncogenes. J Cell Biochem 60: 33-38, 1996.

28. Harvey KF, Pfleger CM and Hariharan IK: The Drosophila Mst ortholog, hippo, restricts growth and cell proliferation and promotes apoptosis. Cell 114: 457-467, 2003.

29. Vogelstein B, Lane D and Levine AJ: Surfing the p53 network. Nature 408: 307-310, 2000.

30. Carr AM, Green MH and Lehmann AR: Checkpoint policing by p53. Nature 359: 486-487, 1992. 
31. Castedo M, Perfettini JL, Roumier T and Kroemer G: Cyclindependent kinase-1: linking apoptosis to cell cycle and mitotic catastrophe. Cell Death Differ 9: 1287-1293, 2002.

32. Olson NE, Graves JD, Shu GL, Ryan EJ and Clark EA: Caspase activity is required for stimulated $\mathrm{B}$ lymphocytes to enter the cell cycle. J Immunol 170: 6065-6072, 2003.

33. Woo M, Hakem R, Furlonger C, Hakem A, Duncan GS, Sasaki T, Bouchard D, Lu L, Wu GE, Paige CJ and Mak TW: Caspase-3 regulates cell cycle in $\mathrm{B}$ cells: a consequence of substrate specificity. Nat Immunol 4: 1016-1022, 2003.

34. McLaughlin B, Hartnett KA, Erhardt JA, Legos JJ, White RF, Barone FC and Aizenman E: Caspase 3 activation is essential for neuroprotection in preconditioning. Proc Natl Acad Sci USA 100: 715-720, 2003.
35. Heenen M, Achten G and Galand P: Cell cycle time of normal epidermis, psoriasis and some epidermal tumors. Ann Dermatol Venereol 106: 131-139, 1979.

36. Tsurusawa M, Aoyama M, Saeki K and Fujimoto T: Cell cycle kinetics in childhood acute leukemia studied with in vitro bromodeoxyuridine labeling, Ki67-reactivity, and flow cytometry. Leukemia 9: 1921-1925, 1995.

37. Preisler HD, Raza A, Gopal V, Ahmad S and Bokhari J: Distribution of cell cycle times amongst the leukemia cells within individual patients with acute myelogenous leukemia. Leuk Res 19: 693-698, 1995. 